\title{
Impact of Nisoldipine Crystal Morphology on its Biopharmaceutical \\ Properties: A Layer Docking Assisted Study \\ Dinesh Kumar ${ }^{\text {a }}$, Rajesh Thipparaboina ${ }^{\text {a }}$, Nalini R Shastri ${ }^{\text {a, }}$,
}

${ }^{a}$ National Institute of Pharmaceutical Education \& Research (NIPER), Hyderabad, India

*Corresponding author. Nalini R. Shastri

Tel. +91-040-23423749

Fax. $+91-040-23073751$

E-mail: nalini.niperhyd@gov.in, svcphod@yahoo.co.in

Address: Department of Pharmaceutics, NIPER (National Institute of Pharmaceutical

Education \& Research), Balanagar, Hyderabad, India, Pin Code - 500037

\section{SUPPORTING INFORMATION}

Supplementary table 1. \% Total facet area by BFDH model, Morphology growth model (MG) and Equilibrium morphology model (EM)

\begin{tabular}{|c|c|c|c|c|}
\hline & \multicolumn{4}{|c|}{$\%$ Total facet area (TFA) } \\
\hline $\mathrm{Hkl}$ & $\mathrm{d}_{\mathrm{hkl}}$ & BFDH & MG & EM \\
\hline$\left\{\begin{array}{llll}0 & 1 & 1\end{array}\right\}$ & 9.34 & 41.07 & 31.24 & 10.15 \\
\hline$\left\{\begin{array}{lll}1 & 0 & -1\end{array}\right\}$ & 8.82 & 12.93 & 25.32 & 18.83 \\
\hline$\left\{\begin{array}{lll}1 & 1 & 0\end{array}\right\}$ & 8.73 & 32.62 & 15.92 & 3.83 \\
\hline$\left\{\begin{array}{llll}0 & 2 & 0\end{array}\right\}$ & 7.82 & 7.60 & 10.93 & 13.91 \\
\hline$\left\{\begin{array}{lll}1 & 1 & -1\end{array}\right\}$ & 7.68 & 2.97 & 4.91 & 12.83 \\
\hline$\left\{\begin{array}{lll}1 & 0 & 1\end{array}\right\}$ & 7.08 & 2.78 & 10.32 & 13.49 \\
\hline
\end{tabular}

Supplementary table 2. Surface chemistry of NSL facets

\begin{tabular}{|c|c|c|c|c|}
\hline \multicolumn{5}{|c|}{ Number of functional groups exposed to crystal surface } \\
\hline$\overline{\mathrm{Hkl}}$ & $\mathrm{CH}_{3}$ & $\mathrm{CO} / \mathrm{COO}$ & $\mathrm{NOO}$ & Aromatic ring \\
\hline$\{011\}$ & $\overline{4}$ & 1 & & \\
\hline $\begin{array}{l}\left\{\begin{array}{lll}1 & 0 & -1\end{array}\right\} \\
\left.\begin{array}{llll}1 & 1 & 0\end{array}\right\}\end{array}$ & 4 & & 1 & $\begin{array}{l}2 \\
1\end{array}$ \\
\hline$\{020\}$ & 2 & 1 & & \\
\hline$\{11-1\}$ & 5 & 1 & & \\
\hline
\end{tabular}


Supplementary table 3. Particle size distribution of NSL crystals

\begin{tabular}{llll}
\hline & $\mathbf{D}_{\mathbf{1 0}}(\boldsymbol{\mu m})$ & $\mathbf{D}_{\mathbf{5 0}}(\boldsymbol{\mu m})$ & $\mathbf{D}_{\mathbf{9 0}}(\boldsymbol{\mu m})$ \\
\hline NSL-M & 232.32 & 309.19 & 401.42 \\
NSL-AI & 240.25 & 294.54 & 390.24 \\
\hline
\end{tabular}

Supplementary table 4 Dissolution parameters of plain NSL and re-crystallized NSL

\begin{tabular}{llll}
\hline & DE $_{\mathbf{6 0}}$ & $\mathbf{D E}_{\mathbf{1 8 0}}$ & $\mathbf{D E}_{\mathbf{3 6 0}}$ \\
\hline NSL & 27.8 & 41.4 & 48.2 \\
NSL-M & 35.8 & 52.7 & 60.9 \\
NSL-AI & 30.8 & 45.2 & 51.4 \\
\hline
\end{tabular}
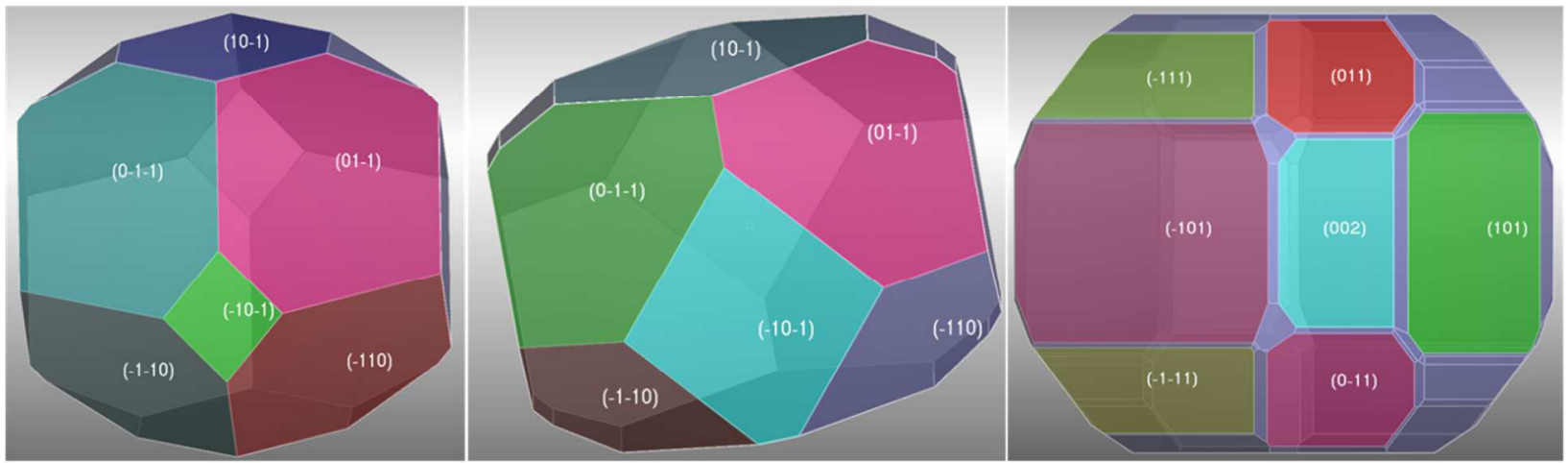

Supplementary figure 1. Simulated crystal habit by a. BFDH, b. Growth morphology (GM), c. Equilibrium morphology (EM) 

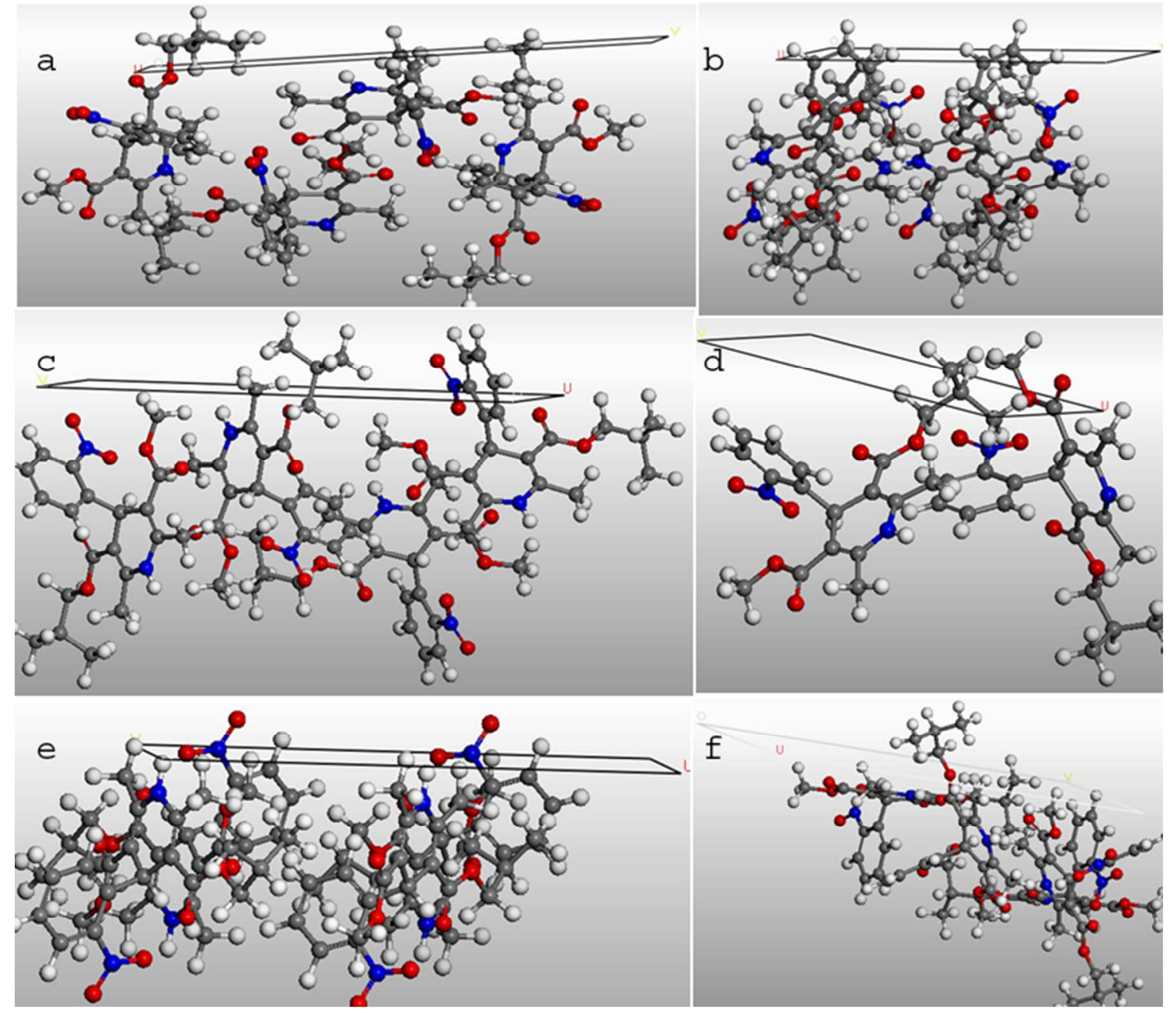

Supplementary figure 2. Crystal slices of different facets of NSL exhibiting the presence of

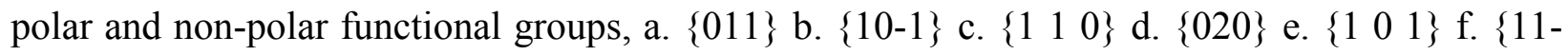
$1\}$ 


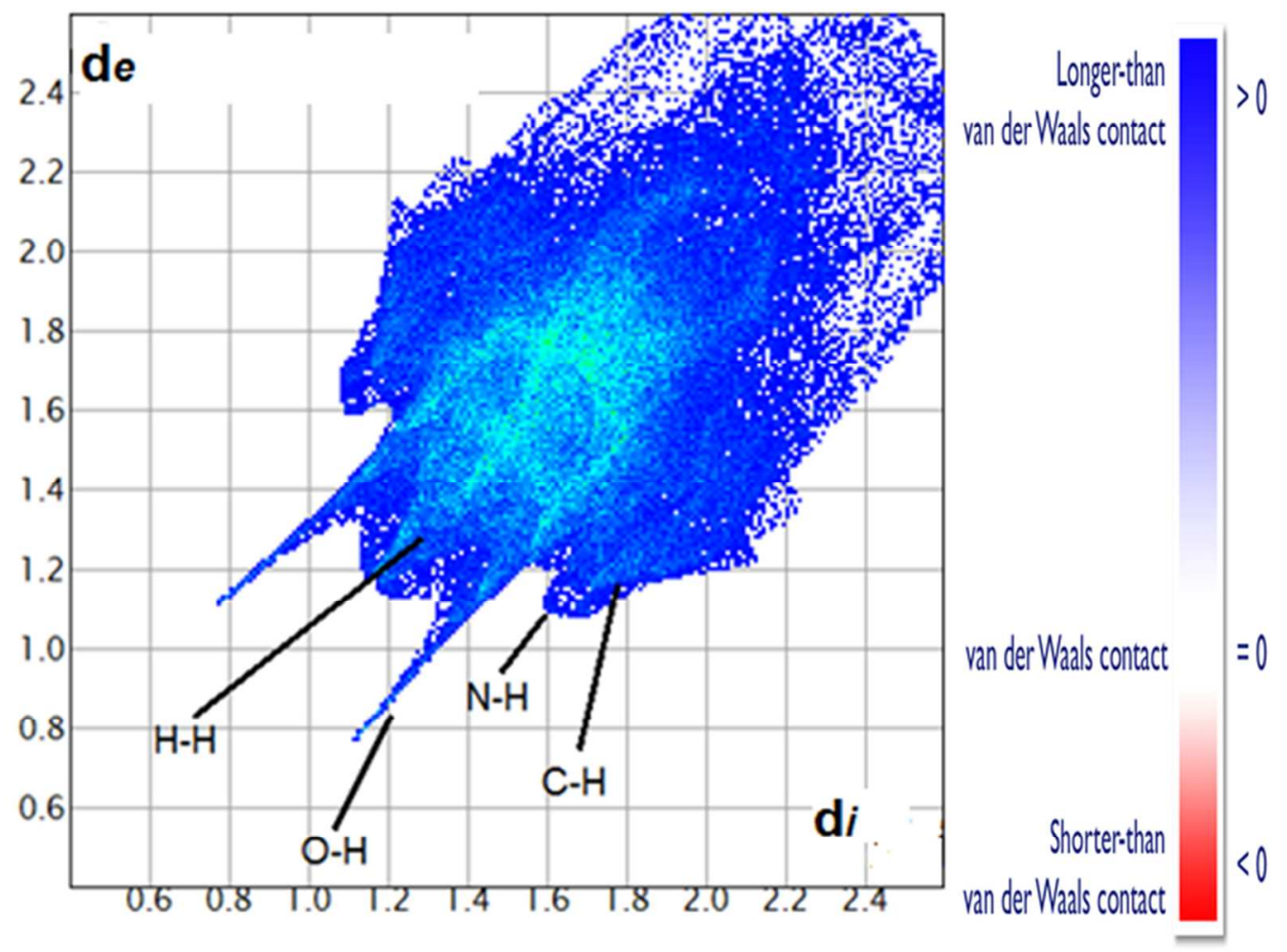

Supplementary figure 3. Fingerprint plots generated from Hirshfeld surface analysis of the NSL (CSD code 7207563 )

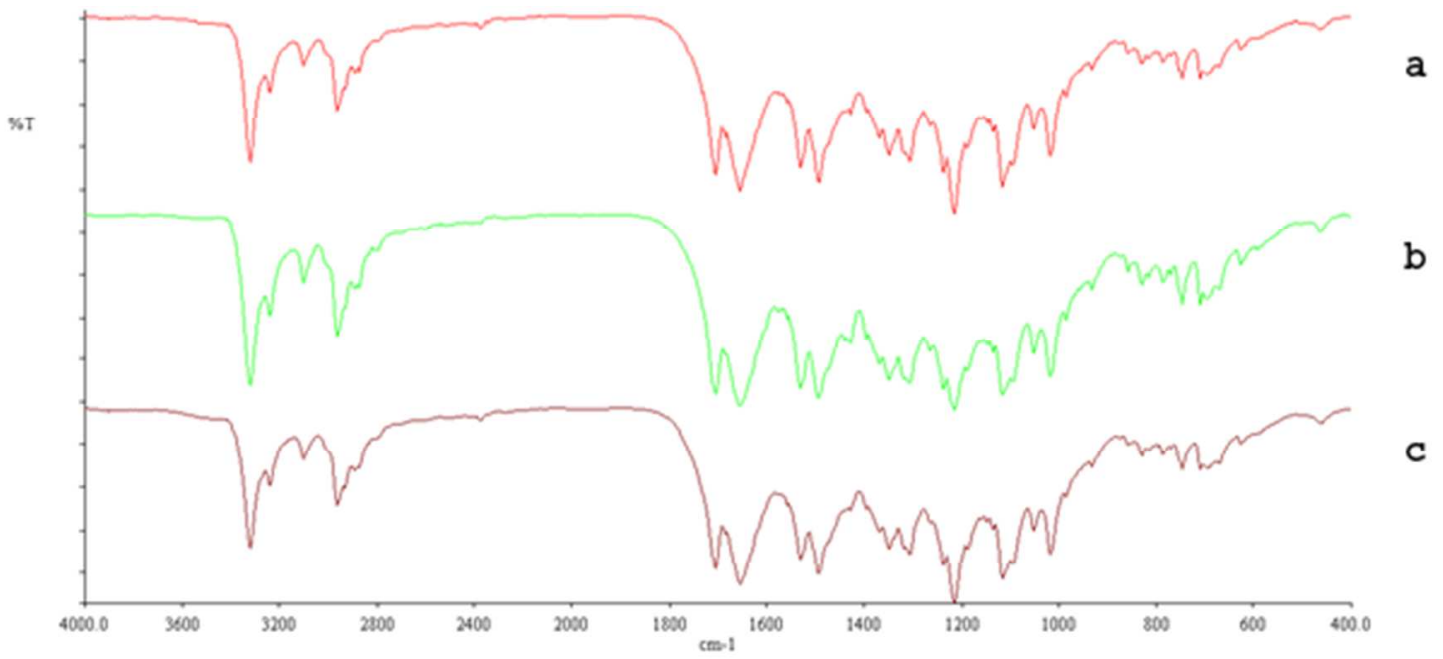

Supplementary figure 4. FTIR of NSL crystals a. NSL, b. NSL-M, c. NSL-AI 


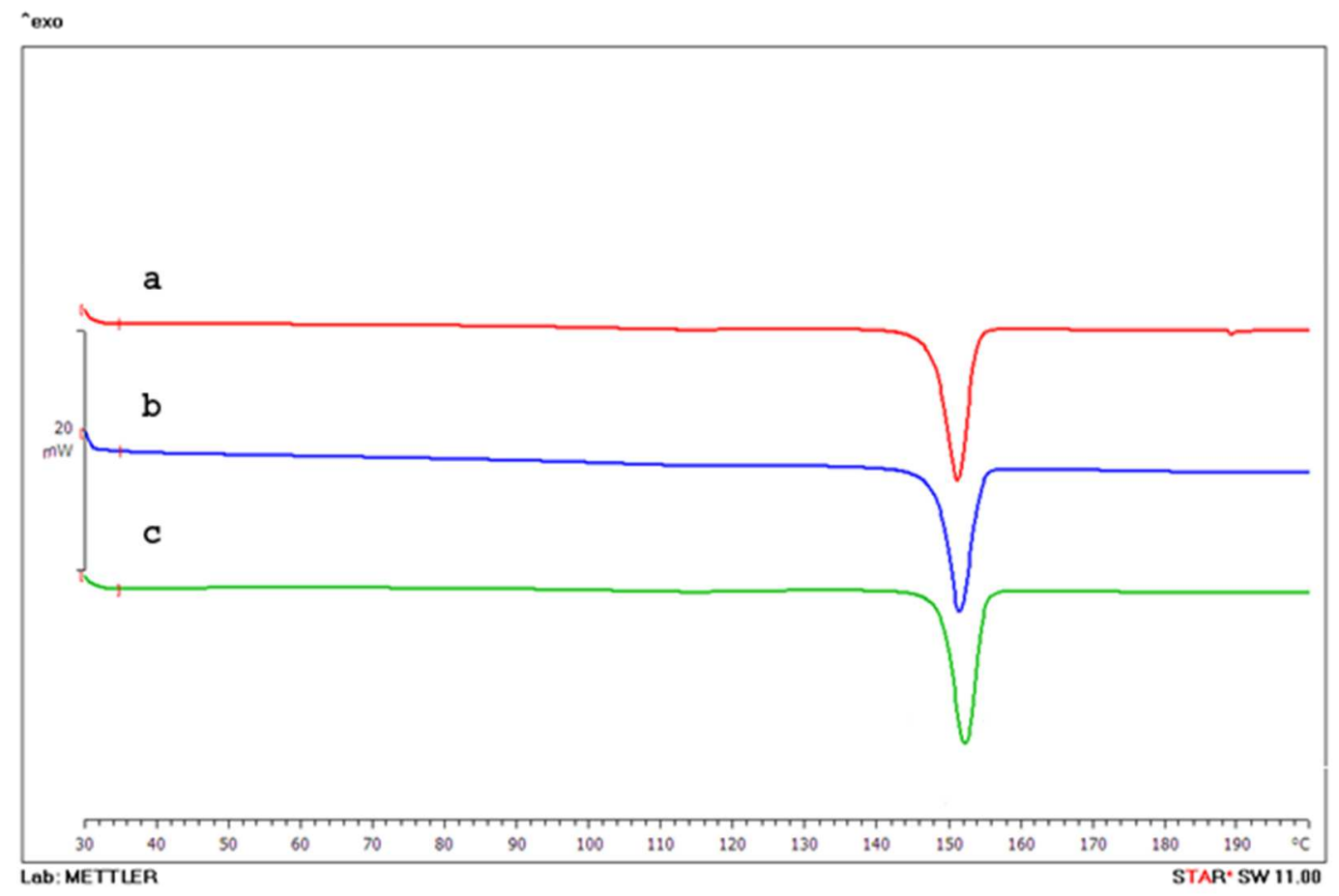

Supplementary figure 5. DSC of NSL crystals a. NSL, b. NSL-M, c. NSL-AI

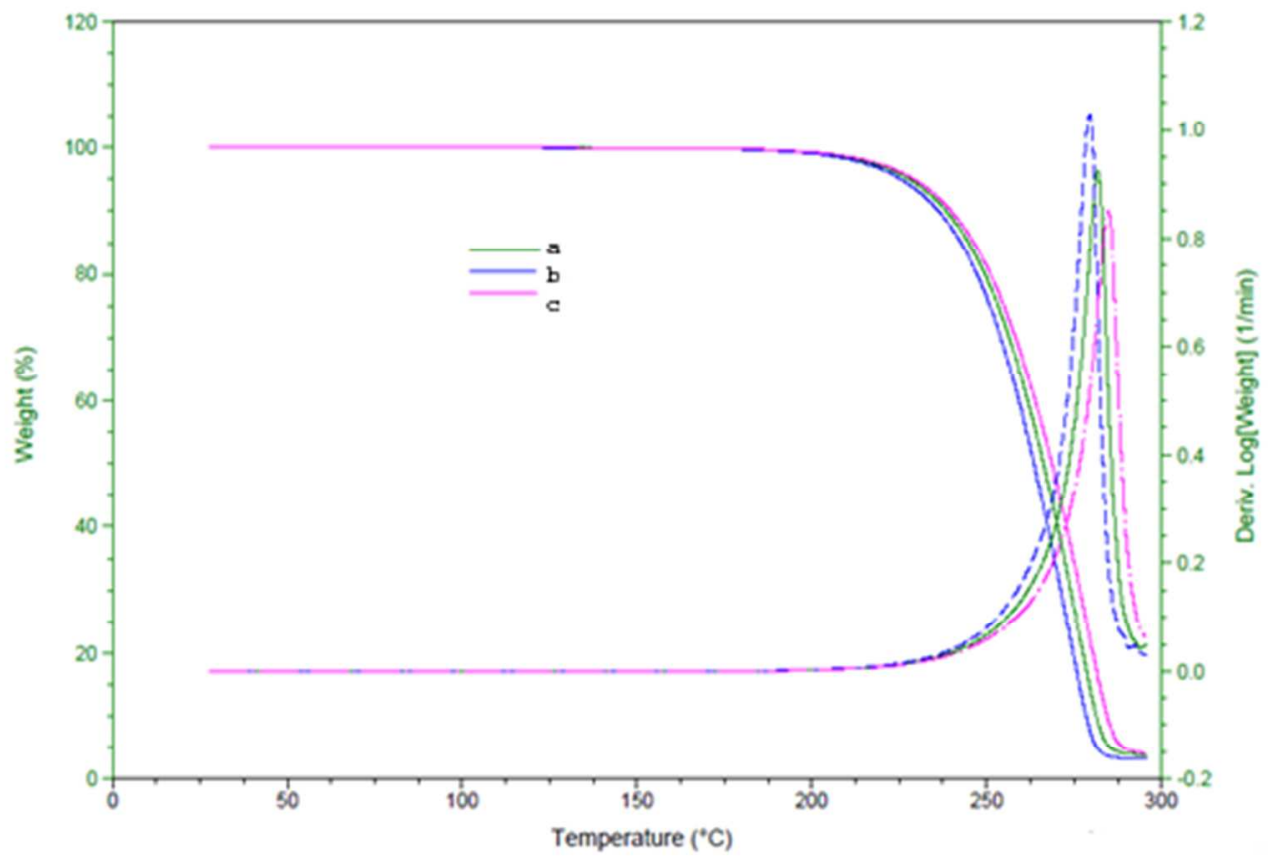

Supplementary figure 6. TGA of NSL crystals a. NSL, b. NSL-M, c. NSL-AI 


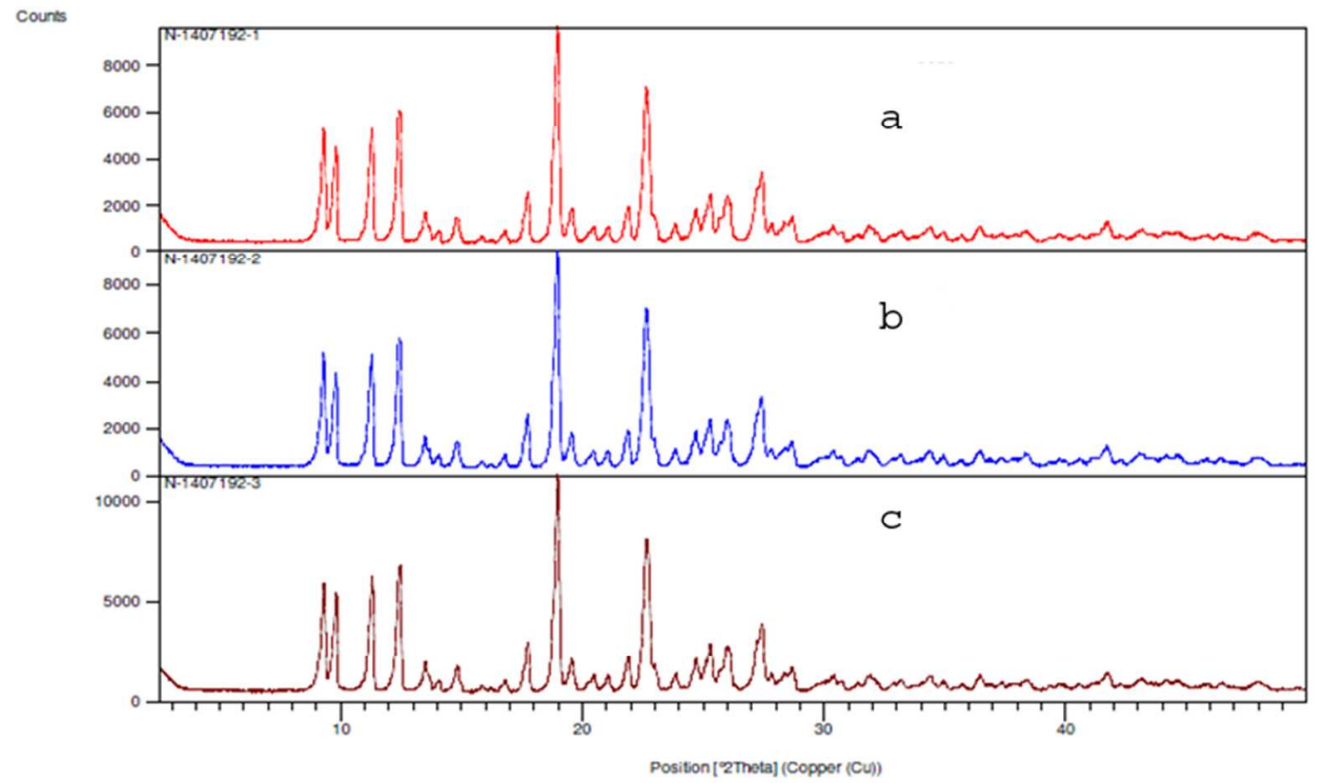

Supplementary figure 7. PXRD of NSL crystals a. NSL, b. NSL-M, c. NSL-AI 